\title{
Convergence behavior of variational perturbation expansion- A method for locating Bender-Wu singularities
}

\author{
H. Kleinert ${ }^{1}$ and W. Janke ${ }^{2}$ \\ ${ }^{1}$ Institut für Theoretische Physik, Freie Universität Berlin, Arnimallee 14, 14195 Berlin, Germany \\ ${ }^{2}$ Institut für Physik, Johannes Gutenberg-Universität Mainz, Staudinger Weg 7, 55099 Mainz, Germany
}

(August 13, 2018)

\begin{abstract}
Variational perturbation expansions have recently been used to calculate directly the strongcoupling expansion coefficients of the anharmonic oscillator. The convergence is exponentially fast with superimposed oscillations, as recently observed empirically by the authors. In this note, the observed behavior is explained and used to determine accurately the magnitude and phase of the leading Bender-Wu singularity which is responsible for the finite convergence radius in the complex coupling constant plane.
\end{abstract}

Variational perturbation theory yields uniformly and exponentially fast converging expansions for many quantum mechanical systems [1 3]. The convergence was proved for the anharmonic integral and the quantum mechanical anharmonic oscillator in several papers for a finite coupling strength $g$ of the anharmonic term 何. Recently, this theory has been adapted to calculating the coefficients of strong-coupling expansions [5]. For the ground-state energy of the anharmonic oscillator, the first 23 coefficients of the series

$$
E^{(0)}=\left(\frac{g}{4}\right)^{1 / 3}\left[\alpha_{0}+\alpha_{1}\left(\frac{g}{4 \omega^{3}}\right)^{-2 / 3}+\alpha_{2}\left(\frac{g}{4 \omega^{3}}\right)^{-4 / 3}+\ldots\right]
$$

were calculated with 20 digits precision. Thereby an interesting phenomenon was observed: the approach to the asymptotic values of $\alpha_{n}$. is exponentially fast with periodic modulations, as shown in Figs. 1 and 2 calling for a revision of the above-quoted proofs which do not apply to the strong-coupling limit $g \rightarrow \infty$. The purpose of this note is to explain the observed behavior theoretically and to show that it leads to a rather precise estimate of the largest Bender-Wu singularities in the complex- $g$-plane which determine the convergence radius of the strong-coupling expansion.

The potential of the anharmonic oscillator is

$$
V(x)=\frac{\omega^{2}}{2} x^{2}+\frac{g}{4} x^{4} \quad\left(\omega^{2}, g>0\right) .
$$

The Rayleigh-Schrödinger perturbation theory yields a power-series expansion

$$
E^{(0)}(g)=\omega \sum_{l=0}^{\infty} E_{l}^{(0)}\left(\frac{g / 4}{\omega^{3}}\right)^{l},
$$

where $E_{l}^{(0)}$ are rational numbers $1 / 2,3 / 4,-21 / 8,333 / 16,-30885 / 128, \ldots$, obtained from the recursion relations of Bender and $\mathrm{Wu}[6]$.

As is well known, the series (3) cannot be used for an evaluation of the energy since it has a zero radius of convergence due to the factorial growth of the coefficients $E_{l}^{(0)}$. The recently developed variational perturbation theory [2] converts the divergent series (3) into an exponentially fast convergent one. The procedure goes as follows (see Section 5.13 of Refs. [2]). First, the harmonic term of the potential is split into a new harmonic term with a trial frequency $\Omega$, and a remainder:

$$
\frac{\omega^{2}}{2} x^{2}=\frac{\Omega^{2}}{2} x^{2}+\left(\frac{\omega^{2}}{2}-\frac{\Omega^{2}}{2}\right) x^{2}
$$

After rewriting

$$
V(x)=\frac{\Omega^{2}}{2} x^{2}+\frac{g}{4}\left(-2 \sigma x^{2} / \Omega+x^{4}\right),
$$

we perform a perturbation expansion in powers of $g$ at a fixed $\sigma=\Omega\left(\Omega^{2}-\omega^{2}\right) / g$, 


$$
E_{N}(g, \sigma)=\Omega \sum_{l=0}^{N} \varepsilon_{l}^{(0)}(\sigma)\left(\frac{\hat{g}}{4}\right)^{l},
$$

where $\hat{g} \equiv g / \Omega^{3}$ is a dimensionless reduced coupling constant.

The calculation of the new series up to a specific order $N$ requires only little work, being easily obtained from the ordinary perturbation series (3) by replacing $\omega$ by $\sqrt{\Omega^{2}-g \sigma / \Omega}$, and by reexpanding (3) in powers of $g$ up to the $N$ th order. This yields the reexpansion coefficients

$$
\varepsilon_{l}^{(0)}(\sigma)=\sum_{j=0}^{l} E_{j}^{(0)}\left(\begin{array}{c}
(1-3 j) / 2 \\
l-j
\end{array}\right)(-4 \sigma)^{l-j} .
$$

The truncated power series $W_{N}(g, \Omega) \equiv E_{N}^{(0)}(g, \sigma)$ is certainly independent of $\Omega$ in the limit $N \rightarrow \infty$. At any finite order, however, it does depend on $\Omega$ via $\sigma$, the approximation having its fastest speed of convergence where it depends least on $\Omega$. If we denote the order-dependent optimal value of $\Omega$ by $\Omega_{N}$, the quantity $W_{N}\left(g, \Omega_{N}\right)$ is the new approximation to $E(g)$ 7. Introducing the reduced frequency $\hat{\omega}=\omega / \Omega$, the approximation can be written as

$$
W_{N}=(g / \hat{g})^{1 / 3} w_{N}\left(\hat{g}, \hat{\omega}^{2}\right) .
$$

From the approximate energies $W_{N}$ it is easy to derive simple formulas for the coefficients of the strong-coupling expansion. We simply expand the function $w_{N}\left(\hat{g}, \hat{\omega}^{2}\right)$ in powers of $\hat{\omega}^{2}=\left(g / \omega^{3}\right)^{-2 / 3} \hat{g}^{2 / 3}$ and find

$$
W_{N}=(g / 4)^{1 / 3}\left[\alpha_{0}+\alpha_{1}\left(\frac{g / 4}{\omega^{3}}\right)^{-2 / 3}+\alpha_{2}\left(\frac{g / 4}{\omega^{3}}\right)^{-4 / 3}+\ldots\right],
$$

with the coefficients

$$
\alpha_{n}=\frac{1}{n !} w_{N}^{(n)}(\hat{g}, 0)(\hat{g} / 4)^{(2 n-1) / 3} .
$$

Here $w_{N}^{(n)}(\hat{g}, 0)$ denotes the $n$ 'th derivative of $w_{N}\left(\hat{g}, \hat{\omega}^{2}\right)$ with respect to $\hat{\omega}^{2}$ at $\hat{\omega}^{2}=0$, which can easily be calculated using (7) [5],

$$
\frac{1}{n !} w_{N}^{(n)}(\hat{g}, 0)=\sum_{l=0}^{N}(-1)^{l+n} \sum_{j=0}^{l-n} E_{j}^{(0)}\left(\begin{array}{c}
(1-3 j) / 2 \\
l-j
\end{array}\right)\left(\begin{array}{c}
l-j \\
n
\end{array}\right)(-\hat{g} / 4)^{j} .
$$

The optimal value of $\Omega_{N}$ has the $N$-dependence (see Ref. [2] and the first of Refs. [3])

$$
\Omega_{N}^{3}=\operatorname{gcN}\left(1+6.85 / N^{2 / 3}\right)
$$

where the coefficient $c$ is $c=0.186047272 \ldots$. With this $\Omega_{N}$, we obtain the exponentially fast approach to the exact limit as shown in Figs. 1 and 2. The exponential falloff is modulated by oscillations.

To explain this behavior we recall that the ground state energy satisfies the subtracted dispersion relation

$$
E^{(0)}(g)=\frac{\omega}{2}+\frac{g}{2 \pi i} \int_{0}^{-\infty} \frac{d g^{\prime}}{g^{\prime}} \frac{\operatorname{disc} E^{(0)}\left(g^{\prime}\right)}{g^{\prime}-g}
$$

where $\operatorname{disc} E^{(0)}\left(g^{\prime}\right)$ denotes the discontinuity across the left-hand cut in the complex $g$-plane (below minus above). An expansion of the integrand in powers of $g$ yields the perturbation series (3). The reexpanded series (6) is obtained from (3) by the above replacement of $\omega \longrightarrow \Omega(1-\sigma \hat{g})^{1 / 2}$ and a reexpansion in powers of $g$.

There is a simple way of obtaining the same reexpansion from the dispersion relation (13). Introducing the dimensionless coupling constant $\bar{g} \equiv g / \omega^{3}$, above replacement amounts to

$$
\bar{g} \longrightarrow \tilde{g}(\hat{g}) \equiv \frac{\hat{g}}{(1-\sigma \hat{g})^{3 / 2}} .
$$

Since Eq. (13) represents an energy, it can be written as $\omega$ times a dimensionless function $\bar{E}^{(0)}(\bar{g})$. Apart from the replacement (14) in the argument, it receives an overall factor $\Omega / \omega=(1-\sigma \hat{g})^{1 / 2}$. If we introduce the reduced energy 
$\hat{E}(\hat{g}) \equiv E(g) / \Omega$, which depends only on the reduced coupling constant $\hat{g}$, the dispersion relation (13) for $E^{(0)}(g)$ turns into

$$
\hat{E}^{(0)}(g)=(1-\sigma \hat{g})^{1 / 2}\left[\frac{1}{2}+\frac{\tilde{g}(\hat{g})}{2 \pi i} \int_{0}^{-\infty} \frac{d \bar{g}^{\prime}}{\bar{g}^{\prime}} \frac{\operatorname{disc} \bar{E}^{(0)}\left(\bar{g}^{\prime}\right)}{\bar{g}^{\prime}-\tilde{g}(\hat{g})}\right] .
$$

The resummed perturbation series is obtained from this by an expansion in powers of $\hat{g} / 4$ up to order $N$.

Note that only the truncation of the expansion causes a difference between the two expressions (13) and (15), since $\bar{g}$ and $\tilde{g}$ are the same numbers, as can be verified by inserting $\hat{g}=g / \Omega^{3}$ and $\sigma$ into the right-hand side of (14).

To find the reexpansion coefficients we observe that the expression (15) satisfies a dispersion relation in the complex $\hat{g}$-plane. If $C$ denotes the cuts in this plane and $\operatorname{disc}_{C} E(\hat{g})$ is the discontinuity across these cuts, the dispersion relation reads

$$
\hat{E}^{(0)}(\hat{g})=\frac{1}{2}+\frac{\hat{g}}{2 \pi i} \int_{C} \frac{d \hat{g}^{\prime}}{\hat{g}^{\prime}} \frac{\operatorname{disc}_{C} \hat{E}^{(0)}\left(\hat{g}^{\prime}\right)}{\hat{g}^{\prime}-\hat{g}} .
$$

We have changed the argument of the energy from $\bar{g}$ to $\hat{g}$ since this will be the relevant variable in the sequel.

When expanding the denominator in the integrand in powers of $\hat{g} / 4$, the expansion coefficients $\varepsilon_{l}^{(0)}$ are found to be moment integrals with respect to the inverse coupling constant $1 / \hat{g}$ :

$$
\varepsilon_{k}^{(0)}=\frac{4^{k}}{2 \pi i} \int_{C} \frac{d \hat{g}}{\hat{g}^{k+1}} \operatorname{disc}_{C} \hat{E}^{(0)}(\hat{g}) .
$$

In the complex $\hat{g}$-plane, the integral (15) has in principle cuts along the contours $C_{1}, C_{\overline{1}}, C_{2}, C_{\overline{2}}$, and $C_{3}$, as shown in Fig. 3. The first four cuts are the images of the left-hand cut in the complex $g$-plane; the curve $C_{3}$ is due to the square root of $1-\sigma \hat{g}$ in the mapping (14) and the prefactor of (15).

Let $\bar{D}(\bar{g})$ abbreviate the reduced discontinuity in the original dispersion relation (13):

$$
\bar{D}(\bar{g}) \equiv \operatorname{disc} \bar{E}^{(0)}(\bar{g})=2 i \operatorname{Im} \bar{E}^{(0)}(\bar{g}-i \eta), \quad \bar{g} \leq 0 .
$$

Then the discontinuities across the various cuts are

$$
\begin{aligned}
& \underset{C_{1, \overline{1}, 2, \overline{2}}}{\operatorname{disc}} \hat{E}^{(0)}(\hat{g})=(1-\sigma \hat{g})^{1 / 2} \bar{D}\left(\hat{g}(1-\sigma \hat{g})^{-3 / 2}\right), \\
& \operatorname{disc}_{C_{3}} \hat{E}^{(0)}(\hat{g})=-2 i(\sigma \hat{g}-1)^{1 / 2} \\
& \times\left[\frac{1}{2}-\int_{0}^{\infty} \frac{d \bar{g}^{\prime}}{2 \pi} \frac{\hat{g}(\sigma \hat{g}-1)^{-3 / 2}}{\bar{g}^{\prime 2}+\hat{g}^{2}(\sigma \hat{g}-1)^{-3}} \bar{D}\left(-\bar{g}^{\prime}\right)\right] .
\end{aligned}
$$

For small negative $\bar{g}$, the discontinuity is given by the semiclassical limit (see [2], Chapter 17):

$$
\bar{D}(\bar{g}) \approx-2 i \sqrt{\frac{6}{\pi}} \sqrt{\frac{4}{-3 \bar{g}}} e^{4 / 3 \bar{g}} .
$$

We denote by $\varepsilon_{k}^{(0)}\left(C_{i}\right)$ the contributions of the different cuts to the integral (17) for the coefficients. After inserting (21) into Eq. (19), we obtain from the cut along $C_{1}$ the semiclassical approximation

$$
\varepsilon_{k}^{(0)}\left(C_{1}\right) \approx-24^{k} \int_{C_{1}} \frac{d \hat{g}}{2 \pi} \frac{1}{\hat{g}^{k+1}} \sqrt{\frac{6}{\pi}} \sqrt{-\frac{4(1-\sigma \hat{g})^{5 / 2}}{3 \hat{g}}} e^{4(1-\sigma \hat{g})^{3 / 2} / 3 \hat{g}} .
$$

For the $k$ th term $S_{k}$ of the series this yields an estimate

$$
S_{k} \propto\left[\int_{C_{\gamma}} \frac{d \gamma}{2 \pi} e^{f_{k}(\gamma)}\right](\sigma \hat{g})^{k}
$$

where $f_{k}(\gamma)$ is the function of $\gamma \equiv \sigma \hat{g}$

$$
f_{k}(\gamma)=-\left(k+\frac{3}{2}\right) \log (-\gamma)+\frac{4 \sigma}{3 \gamma}(1-\gamma)^{3 / 2}
$$


For large $k$, the integral may be evaluated via a saddle point approximation. At the extremum, $\gamma \underset{k \rightarrow \infty}{\longrightarrow} \gamma_{k}=-4 \sigma / 3 k$, $f_{k}(\gamma)$ has the value

$$
f_{k} \underset{k \rightarrow \infty}{\longrightarrow} k \log (3 k / 4 e \sigma)-2 \sigma
$$

The constant $-2 \sigma$ in this limiting expression arises when expanding the second term of Eq. (24) into a Taylor series, $(4 \sigma / 3 \gamma)(1-\gamma)^{3 / 2}=4 \sigma / 3 \gamma_{k}-2 \sigma+\ldots$. Only the first two terms survive the large- $k$ limit.

Thus, to leading order in $k$, the $k$ th term of the reexpanded series becomes

$$
S_{k} \propto e^{-2 \sigma}\left(\frac{-3 k}{e}\right)^{k}\left(\frac{\hat{g}}{4}\right)^{k}
$$

The corresponding reexpansion coefficients are

$$
\varepsilon_{k}^{(0)} \propto e^{-2 \sigma} E_{k}^{(0)}
$$

They have the remarkable property of growing in precisely the same manner with $k$ as the initial expansion coefficients $E_{k}^{(0)}$, except for an overall suppression factor $e^{-2 \sigma}$. This property was discussed in Ref. [2].

In order to estimate the convergence of the variational perturbation expansion, we note that $\sigma \hat{g}=1-1 / \Omega^{2}$. For large $\Omega$, this expression is smaller than unity. Hence the powers $(\sigma \hat{g})^{k}$ alone yield a convergent series. An optimal reexpansion of the energy can be achieved by choosing, for a given large maximal order $N$ of the expansion, a parameter $\sigma$ proportional to $N$ :

$$
\sigma \approx \sigma_{N} \equiv c N
$$

Inserting this into (24), we obtain for large $k=N$

$$
f_{N}(\gamma) \approx N\left[-\log (-\gamma)+\frac{4 c}{3 \gamma}(1-\gamma)^{3 / 2}\right]
$$

The extremum of this function lies at

$$
1+\frac{4 c}{3 \gamma}(1-\gamma)^{1 / 2}\left(1+\frac{1}{2} \gamma\right)=0
$$

The constant $c$ is now chosen in such a way that the large exponent proportional to $N$ in the exponential function $e^{f_{N}(\gamma)}$ due to the first term in (29) is canceled by an equally large contribution from the second term, i.e., we require at the extremum

$$
f_{N}(\gamma)=0
$$

The two equations (30) and (31) are solved by

$$
\gamma=-0.242964029973520 \ldots, \quad c=0.186047272987975 \ldots .
$$

In contrast to the extremal $\gamma$ of Eq. (24) which dominates the large- $k$ limit, the extremal $\gamma$ of the present limit, in which $k$ is also large but of the order of $N$, remains finite (the previous estimate holds for $k \gg N$ ). Accordingly, the second term $(4 c / 3 \gamma)(1-\gamma)^{3 / 2}$ in $f_{N}(\gamma)$ contributes in full, not merely via the first two Taylor expansion terms of $(1-\gamma)^{3 / 2}$, as it did in $(25)$.

Since $f_{N}(\gamma)$ vanishes at the extremum, the $N$ th term in the reexpansion has the order of magnitude

$$
S_{N} \propto\left(\sigma_{N} \hat{g}_{N}\right)^{N}=\left(1-\frac{1}{\Omega_{N}^{2}}\right)^{N}
$$

According to 28 , the frequency $\Omega_{N}$ grows for large $N$ like

$$
\Omega_{N} \sim \sigma_{N}^{1 / 3} g^{1 / 3} \sim(c N g)^{1 / 3}
$$

As a consequence, the last term of the series decreases for large $N$ like 


$$
S_{N}\left(C_{1}\right) \propto\left[1-\frac{1}{\left(\sigma_{N} g\right)^{2 / 3}}\right]^{N} \approx e^{-N /(\sigma g)^{2 / 3}} \approx e^{-N^{1 / 3} /(c g)^{2 / 3}}
$$

This estimate does not yet explain the convergence of the variational perturbation expansion in the strong-coupling limit observed in Figs. 1 and 2. For the contribution of the cut $C_{1}$ to $S_{N}$, the derivation of such a behavior requires including a little more information into the estimate. This information is supplied by the empirically observed property, that the best $\Omega_{N}$-values lie for finite $N$ on a curve (see Chapter 5 in [2]):

$$
\sigma_{N} \sim c N\left(1+\frac{6.85}{N^{2 / 3}}\right)
$$

Thus the asymptotic behavior (28) receives, at a finite $N$, a rather large correction. By inserting this $\sigma_{N}$ into $f_{N}(\gamma)$ of (29), we find an extra exponential factor

$$
\begin{aligned}
e^{\Delta f_{N}} & \approx \exp \left[N \frac{4 c}{3} \frac{(1-\gamma)^{3 / 2}}{\gamma} \frac{6.85}{N^{2 / 3}}\right] \\
& =\exp \left[-N \log (-\gamma) \frac{6.85}{N^{2 / 3}}\right] \approx e^{-9.7 N^{1 / 3}} .
\end{aligned}
$$

This reduces the size of the last term due to the cut $S_{N}\left(C_{1}\right)$ in (35) to

$$
S_{N}\left(C_{1}\right) \propto e^{-\left[9.7+(c g)^{-2 / 3}\right] N^{1 / 3}},
$$

which agrees with the convergence seen in Figs. 11 and 2.

Note that there is no need to evaluate the effect of the shift in the extremal value of $\gamma$ caused by the correction term in (36), since this would be of second order in $1 / N^{2 / 3}$.

How about the contributions of the other cuts? For $C_{\overline{1}}$, the integrals in (17) runs from $\hat{g}=-2 / \sigma$ to $-\infty$ and decrease like $(-2 / \sigma)^{-k}$. The associated last term $S_{N}\left(C_{\overline{1}}\right)$ is of the negligible order $e^{-N \log N}$. For the cuts $C_{2, \overline{2}, 3}$, the integrals (17) start at $\hat{g}=1 / \sigma$ and have therefore the leading behavior

$$
\varepsilon_{k}^{(0)}\left(C_{2, \overline{2}, 3}\right) \sim \sigma^{k}
$$

This implies a contribution to the $N$ th term in the reexpansion of the order of

$$
S_{N}\left(C_{2, \overline{2}, 3}\right) \sim(\sigma \hat{g})^{N},
$$

which decreases merely like (35) and does not explain the empirically observed convergence in the strong-coupling limit. As before, an additional information produces a better estimate. The cuts in Fig. 3 do not really reach the point $\sigma \hat{g}=1$. There exists a small circle of radius $\Delta \hat{g}>0$ in which $\hat{E}^{(0)}(\hat{g})$ has no singularities at all. This is a consequence of the fact unused up to this point that the strong-coupling expansion (i1) converges for $g>g_{\mathrm{s}}$. For the reduced energy, this expansion reads:

$$
\hat{E}^{(0)}(\hat{g})=\left(\frac{\hat{g}}{4}\right)^{1 / 3}\left\{\alpha_{0}+\alpha_{1}\left[\frac{\hat{g}}{4} \frac{1}{(1-\sigma \hat{g})^{3 / 2}}\right]^{-2 / 3}+\alpha_{2}\left[\frac{\hat{g}}{4} \frac{1}{(1-\sigma \hat{g})^{3 / 2}}\right]^{-4 / 3}+\ldots\right\} .
$$

The convergence of (1) for $g>g_{\mathrm{s}}$ implies that (41) converges for all $\sigma \hat{g}$ in a neighborhood of the point $\sigma \hat{g}=1$ with a radius

$$
\Delta(\sigma \hat{g}) \sim\left(\frac{\hat{g}}{-\bar{g}_{\mathrm{s}}}\right)^{2 / 3}=\left\{\frac{1}{-\sigma \bar{g}_{\mathrm{s}}}[1+\Delta(\sigma \hat{g})]\right\}^{2 / 3}
$$

where $\bar{g}_{\mathrm{s}} \equiv g_{\mathrm{s}} / \omega^{3}$. For large $N, \Delta(\sigma \hat{g})$ goes to zero like $1 /(N|\bar{g} s| c)^{2 / 3}$. Thus the integration contours of the moment integrals $(17)$ for the contributions $\varepsilon_{k}^{(0)}\left(C_{i}\right)$ of the other cuts do not begin at the point $\sigma \hat{g}=1$, but a little distance $\Delta(\sigma \hat{g})$ away from it. This generates an additional suppression factor

$$
(\sigma \hat{g})^{-N} \sim[1+\Delta(\sigma \hat{g})]^{-N} .
$$


Let us set $-\bar{g}_{\mathrm{s}}=\left|\bar{g}_{\mathrm{s}}\right| \exp \left(i \varphi_{\mathrm{s}}\right)$ and $x_{\mathrm{s}} \equiv\left(-\hat{g} / \bar{g}_{\mathrm{s}}\right)^{2 / 3}=-\left|x_{\mathrm{s}}\right| \exp (i \theta)$, and introduce the parameter $a \equiv 1 /\left[\left|\bar{g}_{\mathrm{s}}\right| c\right]^{2 / 3}$. Since there are two complex conjugate contributions we obtain, for large $N$ a last term of the order of

$$
S_{N}\left(C_{2, \overline{2}, 3}\right) \approx e^{-N^{1 / 3} a \cos \theta} \cos \left(N^{1 / 3} a \sin \theta\right) .
$$

By choosing

$$
\left|\bar{g}_{\mathrm{s}}\right| \sim 0.160, \quad \theta \sim-0.467
$$

we obtain the curves shown in Figs. 1 and 5, which agree very well with the observed Figs. 1 and 2. Their envelope has the asymptotic falloff $e^{-9.23 N^{1 / 3}}$.

Let us see how the positions of the largest Bender-Wu singularities compare with what we can extract directly from the strong-coupling series (11) up to order 22 [5]. For a pair of square root singularities at $x_{\mathrm{s}}=-\left|x_{\mathrm{s}}\right| \exp ( \pm i \theta)$, the coefficients of a power series $\sum \alpha_{n} x^{n}$ have the asymptotic ratios $R_{n} \equiv \alpha_{n+1} / \alpha_{n} \sim R_{n}^{\text {as }} \equiv-\cos [(n+1) \theta+$ $\delta] /\left|x_{\mathrm{s}}\right| \cos (n \theta+\delta)$. Plotting the ratios $R_{n}$ for the coefficients $\alpha_{n}$ in Fig. 6 we see that for large $n$, they are well reproduced by $R_{n}^{\text {as }}$ if we choose

$$
\left|x_{\mathrm{s}}\right|=1 / 0.117, \quad \theta=-0.467,
$$

with an irrelevant phase angle $\delta=-0.15$. The angle $\theta$ is in excellent agreement with the value found in (45). From $\left|x_{\mathrm{s}}\right|$ we find $\left|\bar{g}_{\mathrm{s}}\right|=4\left|1 / x_{\mathrm{s}}\right|^{3 / 2}=0.160$, again in excellent agreement with (45).

Note that this convergence radius is compatible with the heuristic convergence of the strong-coupling series up to order 22, as can be seen in Fig. 7 by comparing the series with the exact curve.

While this work was in progress, we received a Genova preprint by R. Guida, K. Konishi, and H. Suzuki hepth/9505084) in which the exponentially fast convergence found in Ref. [5] is proved rigorously by reducing it to the known convergence of the strong-coupling expansion.

[1] H. Kleinert, Phys. Lett. A173, 332 (1993); J. Jaenicke and H. Kleinert, Phys. Lett. A176, 409 (1993); H. Kleinert and H. Meyer, Phys. Lett. A184, 319 (1994);

R. Karrlein and H. Kleinert, Phys. Lett. A187, 133 (1994).

The method is a systematic expansion of the first-order approach of R.P. Feynman and H. Kleinert, Phys. Rev. A34, 5080 (1986) and of R. Giachetti and V. Tognetti, Phys. Rev. Lett. 55, 912 (1985); Int. J. Magn. Mater. 54-57, 861 (1986); R. Giachetti, V. Tognetti, and R. Vaia, Phys. Rev. B33, 7647 (1986).

[2] H. Kleinert, Path Integrals in Quantum Mechanics, Statistics and Polymer Physics, 2nd edition (World Scientific, Singapore, 1995). German readers may use the German edition published with (B.I.-Wissenschaftsverlag, Mannheim, 1993).

[3] As far as energy values and not entire path integrals are concerned, similar expansions have been investigated by R. Seznec and J. Zinn-Justin, J. Math. Phys. 20, 1398 (1979); T. Barnes and G.I. Ghandour, Phys. Rev. D22, 924 (1980); B.S. Shaverdyan and A.G. Usherveridze, Phys. Lett. B123, 316 (1983); H. Mitter and K. Yamazaki, J. Phys. A 17, 1215 (1984);

P.M. Stevenson, Phys. Rev. D30, 1712 (1985); D32, 1389 (1985); P.M. Stevenson and R. Tarrach, Phys. Lett. B176, 436 (1986); A. Okopinska, Phys. Rev. D35, 1835 (1987); D36, 2415 (1987); W. Namgung, P.M. Stevenson, and J.F. Reed, Z. Phys. C45, 47 (1989); U. Ritschel, Phys. Lett. B227, 44 (1989); Z. Phys. C51, 469 (1991); M.H. Thoma, Z. Phys. C44, 343 (1991); I. Stancu and P.M. Stevenson, Phys. Rev. D42, 2710 (1991); R. Tarrach, Phys. Lett. B262, 294 (1991); H. Haugerud and F. Raunda, Phys. Rev. D43, 2736 (1991); A.N. Sissakian, I.L. Solivtosv, and O.Y. Sheychenko, Phys. Lett. B313, 367 (1993).

[4] First convergence proofs by

I.R.C. Buckley, A. Duncan, and H.F. Jones, Phys. Rev. D47, 2554 (1993); C.M. Bender, A. Duncan, and H.F. Jones, Phys. Rev. D49, 4219 (1994); A. Duncan and H.F. Jones, Phys. Rev. D47, 2560 (1993); R. Guida, K. Konishi, and H. Suzuki, Genova preprint GEF-Th-7/1994 (hep-th/9407027); C. Arvanitis, H.F. Jones, and C.S. Parker, Imperial College London preprint 1995 (hep-th/9502386)

did not explain the exponentially fast convergence at strong couplings first found in Ref. [5].

[5] W. Janke and H. Kleinert, Phys.Lett. A 199, 287 (1995), quant-ph/9502018; W. Janke and H. Kleinert, Berlin preprint, 1994, quant-ph/9502019. 
[6] C.M. Bender and T.T. Wu, Phys. Rev. 184, 1231 (1969); Phys. Rev. D7, 1620 (1973).

[7] Note that our approximation is far more accurate than the one used in many papers on the acceleration of convergence of perturbation theory such as

Yamazaki, J. Phys. A17, 345 (1984). The approximations in these papers are reproduced if we use, for all $N, \Omega=\Omega 1$ as a variational frequency. 


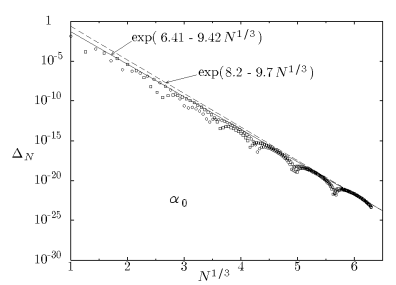

FIG. 1. Exponentially fast convergence of the $N$ th approximants for $\alpha_{0}$ to the exact values. The plotted quantity is $\left.\Delta_{N} \equiv \mid\left(\alpha_{0}\right)_{N}\right)-\alpha_{0} \mid$

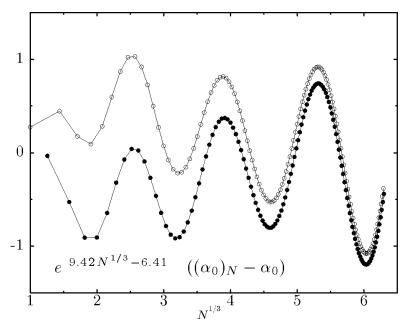

FIG. 2. Oscillatory behavior around the asymptotic approach of $\alpha_{0}$ to its exact value as a function of the order $N$ of the approximant (open circles are for odd $N$, filled circles for even $N$ ).

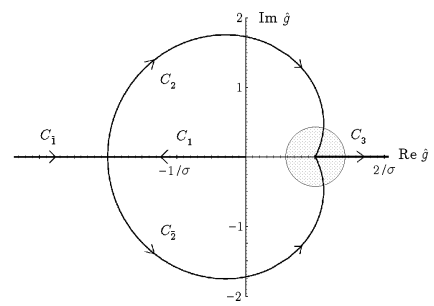


FIG. 3. Cuts in the complex $\hat{g}$-plane whose moments with respect to the inverse coupling constant determine the reexpansion coefficients. The cuts inside the shaded circle happen to be absent due to the convergence of the strong-coupling expansion for $g>g_{\mathrm{s}}$ (from Ref. [2].

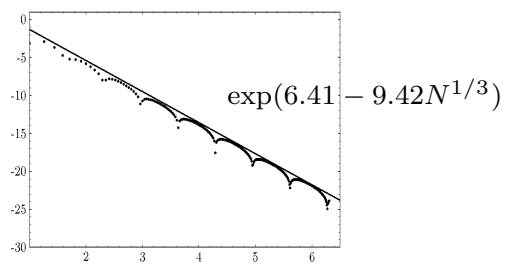

$$
N^{1 / 3}
$$

FIG. 4. Theoretically obtained convergence behavior of the $N$ th approximants $S_{N}$ for $\alpha_{0}$, to be compared with the empirically found behavior in Fig. 1.

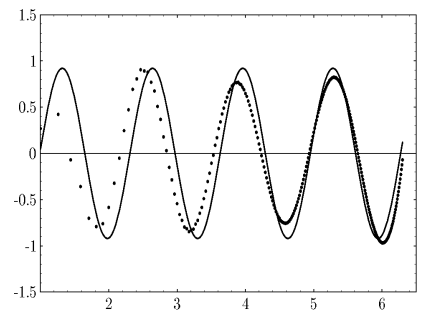

$$
e^{9.23 N^{1 / 3}-5.14} S_{N}
$$

$$
N^{1 / 3}
$$

FIG. 5. Theoretically obtained oscillatory behavior around the asymptotic approach of $\alpha_{0}$ to its exact value as a function of the order $N$ of the approximant, to be compared with the empirically found behavior in Fig. 2, averaged between even and odd orders. 


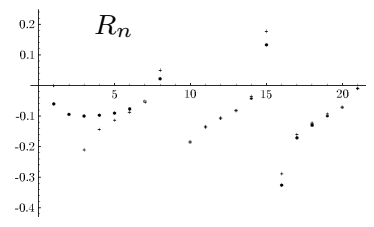

FIG. 6. Comparison of ratios $R_{n}$ between successive expansion coefficients of the strong-coupling expansion (dots) with the ratios $R_{n}^{\text {as }}$ of the exnansinn of a sunerposition of two singularities at $g=0.156 \times \exp ( \pm 0.69)$ (crosses).

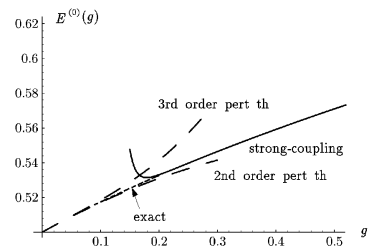

FIG. 7. Strong-Coupling Expansion for the ground-state energy in comparison with the exact values and the perturbative results of 2 nd and 3rd order. The convergence radius in $1 / g$ is larger than $1 / 0.2$. 